\title{
Cross-sectional examination of physical and social contexts of episodes of eating and drinking in a national sample of US adults
}

April $\mathrm{Oh}^{1, *}$, Temitope Erinosho ${ }^{2}$, Genevieve Dunton ${ }^{3}$, Frank M Perna ${ }^{4}$ and

David Berrigan ${ }^{4}$

'Division of Cancer Control and Population Sciences, Clinical Monitoring Research Program Directorate/CMRP, Support to National Cancer Institute, Health Behaviors Research Branch, SAIC-Frederick, Inc., 6130 Executive Blvd, Room 4039, MSC 7335, NCl-Frederick, Frederick, Rockville, MD 21702, USA: ${ }^{2}$ Gillings School of Global Public Health, University of North Carolina at Chapel Hill, Chapel Hill, NC, USA: ${ }^{3}$ Department of Preventive Medicine, University of Southern California, Los Angeles, CA, USA: ${ }^{4}$ Division of Cancer Control and Population Sciences, National Cancer Institute, Rockville, MD, USA

Submitted 21 February 2013: Final revision received 2 November 2013: Accepted 15 November 2013: First published online 15 January 2014

\begin{abstract}
Objective: The current study characterizes associations between physical and social contexts of self-reported primary episodes of eating/drinking and sociodemographic and obesity-related variables in US adults.

Design: Multinomial logistic regression was used to analyse a nationally representative sample of adults from the 2006-2008 American Time Use Survey. Models identifying physical (where) and social (whom) contexts of primary eating/drinking episodes at the population level, controlling for demographic characteristics, weight status and time of eating, were conducted.

Setting: USA.

Subjects: A nationally representative sample of US adults ( $n$ 21315).

Results: Eating/drinking with immediate family was positively associated with age $(\mathrm{OR}=1 \cdot 15(95 \%$ CI $1 \cdot 04,1 \cdot 27)$ to $1 \cdot 23(95 \%$ CI $1 \cdot 09,1 \cdot 39))$, education level $(\mathrm{OR}=1 \cdot 16(95 \%$ CI $1 \cdot 03,1 \cdot 30)$ to $1 \cdot 36(95 \%$ CI $1 \cdot 21,1 \cdot 54))$, obesity (OR $=1 \cdot 13$ $(95 \%$ CI $1 \cdot 04,1 \cdot 22))$, children in the household (OR $=3 \cdot 39(95 \%$ CI 3.14, 3.66)) and time of day $(\mathrm{OR}=1.70(95 \%$ CI $1 \cdot 39,2 \cdot 07)$ to 5.73 (95\% CI $4 \cdot 70,6 \cdot 99))$. Eating in the workplace was negatively associated with female gender $(\mathrm{OR}=0.65(95 \% \mathrm{CI} 0 \cdot 60,0 \cdot 70))$ and children in the household (OR $=0.90(95 \%$ CI $0 \cdot 83,0 \cdot 98)$ ), while positively associated with non-white status (OR $=1 \cdot 14$ (95\% CI $1 \cdot 01,1 \cdot 29)$ to $1 \cdot 47(95 \%$ CI $1 \cdot 32,1 \cdot 65))$ and time of day $(\mathrm{OR}=0 \cdot 25(95 \%$ CI $0 \cdot 28,0 \cdot 30)$ to $5 \cdot 65(95 \%$ CI $4 \cdot 66,6 \cdot 85))$. Women $(\mathrm{OR}=0 \cdot 80(95 \%$ CI $0 \cdot 74$, $0 \cdot 86)$ ), those aged $>34$ years $(\mathrm{OR}=0.48(95 \% \mathrm{CI} 0 \cdot 43,0.54)$ to $0.83(95 \% \mathrm{CI}$ $0.74,0.93))$ and respondents with children $(\mathrm{OR}=0.69(95 \% \mathrm{CI} 0.63,0.75))$ were less likely to eat in a restaurant/bar/retail than at home. Overweight and obese respondents had a greater odds of reporting an episode of eating in social situations $v$. alone (e.g. immediate family and extended family; $\mathrm{OR}=1 \cdot 13$ (95\% CI $1 \cdot 04,1 \cdot 22)$ ) and episodes occurring in restaurant/bar/retail locations $(\mathrm{OR}=1 \cdot 12(95 \% \mathrm{CI} 1 \cdot 03,1 \cdot 23)$ to $1 \cdot 14(95 \% \mathrm{CI} 1 \cdot 05,1 \cdot 24))$.

Conclusions: Findings underscore the multidimensional nature of describing eating/drinking episodes. Social and physical contexts for eating/drinking and their demographic correlates suggest opportunities for tailoring interventions related to diet and may inform intervention targeting and scope.
\end{abstract}

Keywords Eating episodes Social context Physical context Eating behaviours
Understanding eating behaviours has significant implications for population health promotion and prevention of chronic diseases, including obesity, heart disease and some cancers ${ }^{(1-3)}$. Most eating behaviour research has focused on individual characteristics and physiological and psychological determinants of eating ${ }^{(4,5)}$. Recent emphasis on ecological models supports a continued expansion of eating behaviour research to include the study of physical and social contexts in order to better account for patterns of food intake, eating routines and food choice ${ }^{(6)}$.

An episode of eating/drinking is a singular occurrence of eating/drinking behaviour. A comprehensive characterization of eating behaviours includes several dimensions, including 
the temporal pattern of intake, as well as the social (who are you with?) and physical (where are you?) context of eating $^{(7,8)}$. Contexts $^{(9)}$ are factors that are external to the person and food consumed, but are elements that are known to be associated with eating behaviours ${ }^{(9)}$. Food choice theorists argue these contexts of eating interact to influence daily routines of eating ${ }^{(10,11)}$, traditional and socio-cultural meanings of 'meals' (i.e. breakfast, lunch), timing of episodes ${ }^{(10,12)}$, food choices and social norms ${ }^{(13)}$. Context is also associated with food availability and influences food values and preferences (e.g. taste, convenience $)^{(9)}$. However, there are few population studies that describe the physical and social contexts of eating/ drinking episodes and address how they are distributed across population subgroups. Characterizing how eating is distributed across different contexts and demographic groups is an important first step for testing hypotheses about the determinants of eating behaviour and for targeting unhealthy behaviour.

Bisgoni et al.'s framework (2007) on eating/drinking episodes hypothesizes a combination of one or more dimensions of location, time, social setting, mental processes, physical condition and recurrence in predicting episodes of eating and drinking throughout a day ${ }^{(9)}$. As such, information about individual characteristics, households, and location and social dimensions could be used to predict individual or population patterns of eating/drinking episodes. Building on work by Bisgoni et al. to identify dimensions of episodes of eating/ drinking, the current study describes the distribution of eating/drinking episodes across physical and social contexts (two of the multiple dimensions of the framework), and the extent to which such distributions vary by demographic groups, in a national sample of US adults.

In a recent report (2011), Hamrick et al. presented unadjusted results describing the predominant physical and social contexts of eating/drinking episodes among adults using the American Time Use Survey (ATUS) ${ }^{(14)}$. The current study extends past analyses by exploring the effects of adjusting for a number of sociodemographic variables to identify episodes of eating/drinking by major population subgroups within particular contexts. This analysis conceptualizes physical and social contexts as dimensions which can describe episodes of eating and drinking. Because of the documented overlapping and multidimensional nature of describing these episodes, the current study also seeks to explore the potential association between social dimensions of episodes of eating/drinking within physical environments.

\section{Methods}

The present study is a secondary analysis of the 2006-2008 ATUS Eating and Health Module, collected by the US Bureau of Labor Statistics through telephone interviews ${ }^{(15)}$. Households that complete the Current Population Survey are eligible for the ATUS. The sample is nationally representative, including an oversampling of Hispanic and non-Hispanic Black households. More information about the sample and survey methodology can be found at www.bls.gov/tus. The Eating and Health Module collects data on eating and drinking behaviours, specifically, when, where and with whom respondents reported eating/drinking, and any concurrent activities during a $24 \mathrm{~h}$ period.

Respondents over the age of 21 years reporting at least one episode of eating/drinking during the reported $24 \mathrm{~h}$ period in the 2006-2008 ATUS were included in the study. An episode of eating/drinking was any reported eating/ drinking occurring $\geq 1 \mathrm{~min}$ in duration and reported as the primary activity during the $24 \mathrm{~h}$ period. Respondents with an incomplete Eating and Health Module, with missing BMI or who had $\mathrm{BMI} \leq 18.49 \mathrm{~kg} / \mathrm{m}^{2}$, and who responded 'Don't know' or 'Missing' on time spent eating/drinking or physical and social contexts were excluded. Analyses were conducted at the episode level for a total of 65351 primary episodes of eating/drinking, reported by 21315 respondents.

\section{Measures}

\section{Physical context}

The physical context of eating/drinking episodes was defined as 'where' (i.e. the physical space/place) respondents reported they were while eating/drinking. From twenty-five reported categories of physical contexts, responses were collapsed into four major categories based on conceptual definitions, distribution of data and ease of interpretation. The categories included: 'home/ yard', 'workplace', 'restaurant/bar/retail' and 'other place' (place of worship, school, outdoors away from home, library, other place, bank, gym/health club, post office, unspecified place). The distribution for these categories can be found in Appendix 1.

\section{Social context}

The social context of eating/drinking episodes was defined as 'whom' respondents reported they were with when eating/drinking. From twenty reported social context categories, responses were collapsed into five major categories based on conceptual definitions, data distribution and ease of interpretation: 'alone', 'immediate family', 'extended family', 'friends/colleagues/others' and 'multiple categories'. 'Multiple categories' included reported eating/drinking with at least two of the four categories listed above. The distribution for these categories can be found in Appendix 2.

\section{Demographic characteristics}

Demographic characteristics included age, gender, education, employment status and presence/absence of children $<18$ years old in the household. Age was categorized as: 21-34 years, 35-44 years, $45-59$ years and $\geq 60$ years. 
Education was recoded into: 'less than high school', 'highschool graduate/GED', 'some college/associates degree' and 'bachelor's degree or more' (where GED is General Educational Development). Employment was defined as paid employment and categorized as 'full-time' (working $>35$ paid h/week), 'part-time' (working 1-34 paid h/week), 'not employed' (looking for work or not working at a job/ business or unable to work for health reasons/disabled) or 'not in the labour force'. Household children was defined as children aged $<18$ years living within the household.

\section{BMI}

Respondents' self-reported height and weight were used to calculate BMI. BMI was coded as normal weight $\left(18 \cdot 5-24 \cdot 9 \mathrm{~kg} / \mathrm{m}^{2}\right)$, overweight $\left(25 \cdot 0-29 \cdot 9 \mathrm{~kg} / \mathrm{m}^{2}\right)$ and obese $\left(\geq 30 \cdot 0 \mathrm{~kg} / \mathrm{m}^{2}\right)$.

\section{Time}

The start time for reported eating/drinking episodes was used to assign the following time categories based on the distribution of the data: 05.00-10.59 hours, 11.00-15.59 hours, 16.00-22.59 hours and 23.00-04.59 hours.

\section{Analysis}

Multinomial logistic regression models tested whether subpopulations, based on demographic characteristics, were more or less likely to report an eating/drinking episode for physical and social contexts. Odds ratios and predicted marginal proportions (predicted probabilities) were generated $^{(16)}$; employment status was excluded from models examining the physical environment due to correlations with workplace contexts. Associations between social context and episodes of eating/drinking within physical contexts were tested in models including social context as an independent variable, controlling for demographics. Sample weights, to make the sample nationally representative, were applied. Data were analysed using the statistical software package SUDAAN version $9 \cdot 0$.

\section{Results}

The analytical sample was even by gender $(50 \cdot 0 \%$ female) and largely non-Hispanic White (71.7\%; Table 1$)$. Overall, $55.9 \%$ of the sample reported some college/ associates degree or more, $53.4 \%$ were over 44 years old, $36 \cdot 4 \%$ of adults were overweight and $27 \cdot 3 \%$ were obese. Most episodes of primary eating/drinking occurred in the home/yard (71.8\%), with the remainder largely at work (13.6\%) or commercial venues (11.6\%; Table 2). Most episodes of eating/drinking occurred with the immediate family or alone $(70.9 \%$; Table 2$)$.

Regression models of associations between demographic variables and physical contexts of eating/drinking revealed a complex set of patterns (Table 3). We present

Table 1 Demographic characteristics of adult respondents, American Time Use Survey, USA, 2006-2008

\begin{tabular}{|c|c|c|c|}
\hline & $\begin{array}{l}\text { Sample size } \\
(n 21315)\end{array}$ & Weighted \% & SE \\
\hline \multicolumn{4}{|l|}{ Gender } \\
\hline Female & 11914 & $50 \cdot 0$ & $0 \cdot 1$ \\
\hline \multicolumn{4}{|l|}{ Age (years) } \\
\hline $21-34$ & 4535 & $26 \cdot 0$ & $0 \cdot 2$ \\
\hline $45-59$ & 6214 & $29 \cdot 4$ & $0 \cdot 1$ \\
\hline$\geq 60$ & 5456 & $23 \cdot 9$ & $0 \cdot 1$ \\
\hline \multicolumn{4}{|l|}{ Education completed } \\
\hline Less than high school & 2460 & $12 \cdot 0$ & $0 \cdot 3$ \\
\hline High-school graduate/GED & 5993 & $32 \cdot 1$ & $0 \cdot 3$ \\
\hline Some college/associates degree & 6013 & $26 \cdot 9$ & $0 \cdot 4$ \\
\hline$>185 \%$ of poverty threshold & 14541 & $70 \cdot 0$ & $0 \cdot 4$ \\
\hline Missing & 616 & $3 \cdot 2$ & $0 \cdot 1$ \\
\hline \multicolumn{4}{|l|}{ Race/ethnicity } \\
\hline Non-Hispanic White only & 14953 & $71 \cdot 7$ & $0 \cdot 3$ \\
\hline Non-Hispanic Black only & 2711 & $11 \cdot 4$ & $0 \cdot 1$ \\
\hline Hispanic & 2673 & $12 \cdot 0$ & $0 \cdot 2$ \\
\hline Non-Hispanic API only & 635 & $3 \cdot 2$ & $0 \cdot 2$ \\
\hline Non-Hispanic $\mathrm{Al} / \mathrm{AN}$ only & 101 & 0.5 & $0 \cdot 1$ \\
\hline Non-Hispanic mixed & 242 & $1 \cdot 2$ & $0 \cdot 1$ \\
\hline \multicolumn{4}{|l|}{ Weight status } \\
\hline Normal $\left(B M I=18 \cdot 5-24.9 \mathrm{~kg} / \mathrm{m}^{2}\right)$ & 7457 & $34 \cdot 9$ & $0 \cdot 4$ \\
\hline
\end{tabular}

GED, General Educational Development; API, Asian Pacific Islander; Al, American Indian; AN, Alaskan Native. 
Table 2 Physical and social context of eating and drinking episodes, American Time Use Survey, USA, 2006-2008

\begin{tabular}{|c|c|c|c|c|c|}
\hline \multirow[b]{2}{*}{ Physical context } & \multicolumn{2}{|c|}{ Eating and drinking* } & \multirow[b]{2}{*}{ Social context } & \multicolumn{2}{|c|}{ Eating and drinking* } \\
\hline & $n$ & $\%$ & & $n$ & $\%$ \\
\hline Home/yard & 48796 & $71 \cdot 8$ & Alone & 23655 & 34.5 \\
\hline Workplace & 6625 & $13 \cdot 6$ & Immediate family & 23638 & $36 \cdot 4$ \\
\hline Restaurant/bar/retail & 7849 & $11 \cdot 6$ & Extended family & 3526 & $5 \cdot 8$ \\
\hline \multirow{2}{*}{ Other place } & 2081 & $3 \cdot 0$ & Friends/colleagues/others & 8300 & $14 \cdot 7$ \\
\hline & & & Multiple categories & 6206 & $8 \cdot 6$ \\
\hline Total & 65351 & & Total & 65325 & \\
\hline
\end{tabular}

Further detail on the breakdown for each physical and social context category may be found in Appendix 1 and Appendix 2 , respectively.

${ }^{*}$ Numbers reported are weighted.

Table 3 Odds ratios and $95 \%$ confidence intervals for the odds of eating/drinking episodes reported within physical contexts by sociodemographic characteristics and time, American Time Use Survey, USA, 2006-2008. For this model the adjusted Wald $F=538.5$ $(P<0 \cdot 01)$

\begin{tabular}{|c|c|c|c|c|c|c|}
\hline & \multicolumn{2}{|c|}{ Workplace v. Home/yard } & \multicolumn{2}{|c|}{ Restaurant/bar/retail $v$. Home/yard } & \multicolumn{2}{|c|}{ Other $v$. Home/yard } \\
\hline & OR & $95 \% \mathrm{Cl}$ & OR & $95 \% \mathrm{Cl}$ & OR & $95 \% \mathrm{Cl}$ \\
\hline \multicolumn{7}{|l|}{ Gender } \\
\hline Male (ref.) & $1 \cdot 00$ & $1 \cdot 00,1 \cdot 00$ & 1.00 & $1 \cdot 00,1 \cdot 00$ & 1.00 & $1 \cdot 00,1.00$ \\
\hline Female & 0.65 & $0 \cdot 60,0 \cdot 70$ & $0 \cdot 80$ & $0.74,0.86$ & 0.69 & $0.63,0.75$ \\
\hline \multicolumn{7}{|l|}{ Age (years) } \\
\hline 21-34 (ref.) & 1.00 & $1 \cdot 00,1 \cdot 00$ & 1.00 & $1 \cdot 00,1 \cdot 00$ & 1.00 & $1.00,1.00$ \\
\hline $35-44$ & 0.93 & $0 \cdot 84,1 \cdot 03$ & 0.83 & $0.74,0.93$ & $0 \cdot 72$ & $0.56,0.93$ \\
\hline $45-59$ & 0.91 & $0 \cdot 82,1 \cdot 01$ & 0.66 & $0.58,0.74$ & 0.65 & $0.51,0.84$ \\
\hline$\geq 60$ & $0 \cdot 17$ & $0 \cdot 15,0 \cdot 19$ & 0.48 & $0.43,0.54$ & 0.56 & $0.43,0.73$ \\
\hline \multicolumn{7}{|l|}{ Education } \\
\hline Less than high school (ref.) & 1.00 & $1 \cdot 00,1 \cdot 00$ & $1 \cdot 00$ & $1 \cdot 00,1 \cdot 00$ & 1.00 & $1 \cdot 00,1.00$ \\
\hline High-school graduate/GED & $1 \cdot 17$ & $1 \cdot 00,1 \cdot 38$ & 1.56 & $1 \cdot 34,1 \cdot 82$ & $1 \cdot 25$ & $0.94,1.67$ \\
\hline Some college/associates degree & $1 \cdot 11$ & $0.94,1 \cdot 32$ & 1.93 & $1 \cdot 67,2 \cdot 23$ & $1 \cdot 37$ & $1 \cdot 02,1 \cdot 83$ \\
\hline Bachelor's degree or more & 0.96 & $0 \cdot 81,1 \cdot 13$ & $2 \cdot 07$ & $1 \cdot 79,2 \cdot 41$ & $1 \cdot 75$ & $1 \cdot 31,2 \cdot 34$ \\
\hline \multicolumn{7}{|l|}{ Race/ethnicity } \\
\hline Non-Hispanic White only (ref.) & $1 \cdot 00$ & $1 \cdot 00,1 \cdot 00$ & $1 \cdot 00$ & $1 \cdot 00,1 \cdot 00$ & $1 \cdot 00$ & $1.00,1.00$ \\
\hline Non-Hispanic Black only & $1 \cdot 14$ & $1 \cdot 01,1 \cdot 29$ & 0.56 & $0.48,0.64$ & 0.77 & $0.63,0.95$ \\
\hline Hispanic & $1 \cdot 47$ & $1 \cdot 32,1 \cdot 65$ & 0.79 & $0 \cdot 70,0.90$ & $0 \cdot 71$ & $0.55,0.92$ \\
\hline Non-Hispanic API/AI/AN/mixed & $1 \cdot 29$ & $1 \cdot 07,1.56$ & $0 \cdot 78$ & $0.64,0.95$ & $0 \cdot 77$ & $0.53,1.12$ \\
\hline \multicolumn{7}{|l|}{ Weight status } \\
\hline Normal $\left(B M I=18.5-24.9 \mathrm{~kg} / \mathrm{m}^{2}\right)$ (ref.) & $1 \cdot 00$ & $1 \cdot 00,1 \cdot 00$ & $1 \cdot 00$ & $1 \cdot 00,1 \cdot 00$ & $1 \cdot 00$ & $1.00,1.00$ \\
\hline Overweight $\left(\mathrm{BMI}=25 \cdot 0-29 \cdot 9 \mathrm{~kg} / \mathrm{m}^{2}\right)$ & $1 \cdot 10$ & $0.99,1 \cdot 22$ & $1 \cdot 12$ & $1 \cdot 03,1 \cdot 23$ & $1 \cdot 00$ & $0 \cdot 84,1 \cdot 20$ \\
\hline Obese $\left(\mathrm{BMI} \geq 30 \cdot 0 \mathrm{~kg} / \mathrm{m}^{2}\right)$ & $1 \cdot 10$ & $0.99,1.22$ & $1 \cdot 14$ & $1 \cdot 05,1 \cdot 24$ & $1 \cdot 19$ & $1 \cdot 01,1 \cdot 40$ \\
\hline \multicolumn{7}{|l|}{ Children $<18$ years in household } \\
\hline No (ref.) & $1 \cdot 00$ & $1 \cdot 00,1 \cdot 00$ & $1 \cdot 00$ & $1 \cdot 00,1 \cdot 00$ & $1 \cdot 00$ & $1 \cdot 00,1.00$ \\
\hline Yes & 0.90 & $0.83,0.98$ & 0.69 & $0.63,0.75$ & 0.88 & $0.74,1.05$ \\
\hline \multicolumn{7}{|l|}{ Time } \\
\hline 23.00-04.59 hours (ref.) & 1.00 & $1 \cdot 00,1 \cdot 00$ & 1.00 & $1 \cdot 00,1 \cdot 00$ & 1.00 & $1.00,1.00$ \\
\hline $05.00-10.59$ hours & $1 \cdot 10$ & $0.90,1.33$ & $0 \cdot 85$ & $0.68,1.08$ & $1 \cdot 32$ & $0 \cdot 81,2 \cdot 13$ \\
\hline $11.00-15.59$ hours & $5 \cdot 65$ & $4 \cdot 66,6 \cdot 85$ & $4 \cdot 35$ & $3 \cdot 45,5 \cdot 47$ & 3.90 & $2 \cdot 43,6 \cdot 25$ \\
\hline $16.00-22.59$ hours & $0 \cdot 25$ & $0.28,0.30$ & $1 \cdot 82$ & $1 \cdot 45,2 \cdot 28$ & $1 \cdot 08$ & $0.67,1 \cdot 75$ \\
\hline
\end{tabular}

ref., reference category; GED, General Educational Development; API, Asian Pacific Islander; Al, American Indian; AN, Alaskan Native.

Multinomial logistic regression models performed the regression of physical context (home/yard, workplace, restaurant/bar/retail, other) v. gender, age, education, race/ethnicity, BMI and time.

All variables were significant at $P<0.01$ except weight status.

odds ratios for episodes occurring at the workplace, a restaurant/bar/retail or other location $v$. the home/yard. Eating in the workplace was negatively associated with female gender $(\mathrm{OR}=0.65(95 \% \mathrm{CI} 0.60,0.70))$ and having children in the household ( $\mathrm{OR}=0.90(95 \% \mathrm{CI}$ $0.83,0.98)$ ), while positively associated with non-white status $(\mathrm{OR}=1 \cdot 14(95 \% \mathrm{CI} 1 \cdot 01,1 \cdot 29)$ to $1.47(95 \% \mathrm{CI}$ $1 \cdot 32,1 \cdot 65))$ and time of day $(\mathrm{OR}=0.25(95 \%$ CI $0 \cdot 28$, $0 \cdot 30)$ to $5 \cdot 65(95 \%$ CI $4 \cdot 66,6 \cdot 85))$. Respondents aged $\geq 60$ years were much less likely to eat at the workplace $(\mathrm{OR}=0.17(95 \%$ CI $0.15,0 \cdot 19)) \quad v$. the home/yard. Women $(\mathrm{OR}=0 \cdot 80(95 \% \mathrm{CI} 0 \cdot 74,0 \cdot 86))$, people over 34 years old $(\mathrm{OR}=0.48(95 \% \mathrm{CI} 0 \cdot 43,0.54)$ to $0.83(95 \% \mathrm{CI}$ $0.74,0.93))$, non-whites $(\mathrm{OR}=0.56(95 \%$ CI $0.48,0.64)$ to $0.79(95 \%$ CI $0.70,0.90))$ and respondents with children (OR $=0.69(95 \%$ CI $0.63,0.75))$ were less likely to eat in a restaurant/bar/retail than at home. Eating in a restaurant/bar/retail was more likely in greater than 
Table 4 Odds ratios and $95 \%$ confidence intervals for the odds of eating/drinking episodes reported within social contexts by sociodemographic characteristics and time, American Time Use Survey, USA, 2006-2008. For this model the adjusted Wald $F=357 \cdot 9$ $(P<0 \cdot 01)$

\begin{tabular}{|c|c|c|c|c|c|c|c|c|}
\hline & \multicolumn{2}{|c|}{$\begin{array}{c}\text { Immediate family } v \text {. } \\
\text { Alone }\end{array}$} & \multicolumn{2}{|c|}{$\begin{array}{l}\text { Extended family } v \text {. } \\
\text { Alone }\end{array}$} & \multicolumn{2}{|c|}{$\begin{array}{l}\text { Friends/colleagues/ } \\
\text { others } v \text {. Alone }\end{array}$} & \multicolumn{2}{|c|}{$\begin{array}{c}\text { Multiple categories } v \\
\text { Alone }\end{array}$} \\
\hline & OR & $95 \% \mathrm{Cl}$ & OR & $95 \% \mathrm{Cl}$ & OR & $95 \% \mathrm{Cl}$ & OR & $95 \% \mathrm{Cl}$ \\
\hline \multicolumn{9}{|l|}{ Gender } \\
\hline Male (ref.) & $1 \cdot 00$ & $1 \cdot 00,1 \cdot 00$ & $1 \cdot 00$ & $1 \cdot 00,1 \cdot 00$ & $1 \cdot 00$ & $1 \cdot 00,1 \cdot 00$ & $1 \cdot 00$ & $1 \cdot 00,1 \cdot 00$ \\
\hline Female & $1 \cdot 04$ & $0 \cdot 97,1 \cdot 11$ & $1 \cdot 58$ & $1 \cdot 38,1 \cdot 81$ & 0.98 & $0 \cdot 91,1 \cdot 06$ & $1 \cdot 39$ & $1 \cdot 24,1 \cdot 56$ \\
\hline \multicolumn{9}{|l|}{ Age (years) } \\
\hline 21-34 (ref.) & $1 \cdot 00$ & $1 \cdot 00,1 \cdot 00$ & $1 \cdot 00$ & $1 \cdot 00,1 \cdot 00$ & $1 \cdot 00$ & $1 \cdot 00,1 \cdot 00$ & $1 \cdot 00$ & $1 \cdot 00,1 \cdot 00$ \\
\hline $35-44$ & $1 \cdot 15$ & $1 \cdot 04,1 \cdot 27$ & $0 \cdot 39$ & $0.32,0.47$ & $0 \cdot 66$ & $0.59,0.74$ & $0 \cdot 75$ & $0 \cdot 64,0 \cdot 88$ \\
\hline $45-59$ & $1 \cdot 18$ & $1 \cdot 07,1 \cdot 30$ & 0.35 & $0.30,0.42$ & 0.51 & $0.46,0.58$ & 0.94 & $0 \cdot 79,1 \cdot 10$ \\
\hline$\geq 60$ & $1 \cdot 23$ & $1 \cdot 09,1 \cdot 39$ & $0 \cdot 25$ & $0 \cdot 20,0 \cdot 31$ & $0 \cdot 39$ & $0 \cdot 34,0 \cdot 46$ & 0.93 & $0 \cdot 75,1 \cdot 15$ \\
\hline \multicolumn{9}{|l|}{ Education } \\
\hline Less than high school (ref.) & $1 \cdot 00$ & $1 \cdot 00,1 \cdot 00$ & $1 \cdot 00$ & $1 \cdot 00,1 \cdot 00$ & $1 \cdot 00$ & $1 \cdot 00,1 \cdot 00$ & $1 \cdot 00$ & $1 \cdot 00,1 \cdot 00$ \\
\hline High-school graduate/GED & $1 \cdot 17$ & $1 \cdot 05,1 \cdot 31$ & 0.99 & $0 \cdot 81,1 \cdot 21$ & 0.96 & $0.83,1 \cdot 12$ & $1 \cdot 05$ & $0 \cdot 88,1 \cdot 25$ \\
\hline Some college/associates degree & $1 \cdot 16$ & $1 \cdot 03,1 \cdot 30$ & 0.93 & $0 \cdot 76,1 \cdot 14$ & 0.97 & $0 \cdot 84,1 \cdot 13$ & 0.98 & $0 \cdot 82,1 \cdot 16$ \\
\hline Bachelor's degree or more & $1 \cdot 36$ & $1 \cdot 21,1 \cdot 54$ & 0.62 & $0.51,0.77$ & $0 \cdot 86$ & $0.74,0.99$ & $1 \cdot 07$ & $0 \cdot 90,1 \cdot 27$ \\
\hline \multicolumn{9}{|l|}{ Race/ethnicity } \\
\hline Non-Hispanic White only (ref.) & $1 \cdot 00$ & $1 \cdot 00,1 \cdot 00$ & $1 \cdot 00$ & $1 \cdot 00,1 \cdot 00$ & $1 \cdot 00$ & $1 \cdot 00,1 \cdot 00$ & $1 \cdot 00$ & $1 \cdot 00,1 \cdot 00$ \\
\hline Non-Hispanic Black only & 0.43 & $0.39,0.48$ & $0 \cdot 78$ & $0.67,0.92$ & 0.68 & $0 \cdot 60,0.78$ & $0 \cdot 37$ & $0 \cdot 32,0.44$ \\
\hline Hispanic & $1 \cdot 10$ & $1 \cdot 00,1 \cdot 22$ & $1 \cdot 49$ & $1 \cdot 22,1 \cdot 81$ & $1 \cdot 26$ & $1 \cdot 12,1 \cdot 43$ & $1 \cdot 31$ & $1 \cdot 13,1 \cdot 52$ \\
\hline Non-Hispanic API/AI/AN/mixed & 0.99 & $0 \cdot 85,1 \cdot 16$ & $0 \cdot 88$ & $0 \cdot 64,1 \cdot 23$ & $0 \cdot 82$ & $0.66,1.03$ & $0 \cdot 91$ & $0 \cdot 71,1 \cdot 16$ \\
\hline \multicolumn{9}{|l|}{ Weight status } \\
\hline Normal $\left(\mathrm{BMI}=18.5-24.9 \mathrm{~kg} / \mathrm{m}^{2}\right)$ (ref.) & 1.00 & $1 \cdot 00,1 \cdot 00$ & 1.00 & $1 \cdot 00,1 \cdot 00$ & 1.00 & $1 \cdot 00,1 \cdot 00$ & 1.00 & $1 \cdot 00,1 \cdot 00$ \\
\hline Overweight $\left(\mathrm{BMI}=25 \cdot 0-29 \cdot 9 \mathrm{~kg} / \mathrm{m}^{2}\right)^{\prime}$ & $1 \cdot 11$ & $1 \cdot 03,1 \cdot 20$ & $1 \cdot 13$ & $0.95,1.33$ & $1 \cdot 02$ & $0.93,1.12$ & $1 \cdot 25$ & $1 \cdot 12,1 \cdot 40$ \\
\hline Obese $\left(\mathrm{BMI} \geq 30 \cdot 0 \mathrm{~kg} / \mathrm{m}^{2}\right)$ & $1 \cdot 13$ & $1 \cdot 04,1 \cdot 22$ & $1 \cdot 30$ & $1 \cdot 11,1.53$ & $1 \cdot 00$ & $0.90,1 \cdot 11$ & $1 \cdot 30$ & $1 \cdot 16,1 \cdot 46$ \\
\hline \multicolumn{9}{|l|}{ Children $<18$ years in household } \\
\hline No (ref.) & $1 \cdot 00$ & $1 \cdot 00,1 \cdot 00$ & 1.00 & $1 \cdot 00,1 \cdot 00$ & $1 \cdot 00$ & $1 \cdot 00,1 \cdot 00$ & $1 \cdot 00$ & $1 \cdot 00,1 \cdot 00$ \\
\hline Yes & $3 \cdot 39$ & $3 \cdot 14,3 \cdot 66$ & $0 \cdot 62$ & $0 \cdot 54,0.72$ & $1 \cdot 06$ & $0 \cdot 97,1 \cdot 16$ & $4 \cdot 13$ & $3 \cdot 59,4 \cdot 75$ \\
\hline \multicolumn{9}{|l|}{ Employment } \\
\hline Full-time (ref.) & $1 \cdot 00$ & $1 \cdot 00,1 \cdot 00$ & $1 \cdot 00$ & $1 \cdot 00,1 \cdot 00$ & $1 \cdot 00$ & $1 \cdot 00,1 \cdot 00$ & $1 \cdot 00$ & $1 \cdot 00,1 \cdot 00$ \\
\hline Part-time & $1 \cdot 22$ & $1 \cdot 09,1 \cdot 36$ & $1 \cdot 37$ & $1 \cdot 14,1 \cdot 66$ & $0 \cdot 75$ & $0 \cdot 65,0.86$ & $1 \cdot 13$ & $0 \cdot 97,1 \cdot 31$ \\
\hline Unemployed & $1 \cdot 09$ & $0 \cdot 96,1 \cdot 24$ & $1 \cdot 44$ & $1 \cdot 15,1 \cdot 80$ & $0 \cdot 32$ & $0 \cdot 26,0 \cdot 40$ & $1 \cdot 08$ & $0 \cdot 82,1 \cdot 41$ \\
\hline Not in labour force & $1 \cdot 83$ & $1 \cdot 67,2 \cdot 00$ & $1 \cdot 65$ & $1 \cdot 34,2 \cdot 02$ & 0.44 & $0 \cdot 38,0 \cdot 50$ & 1.57 & $1 \cdot 35,1 \cdot 81$ \\
\hline \multicolumn{9}{|l|}{ Time } \\
\hline 23.00-04.59 hours (ref.) & $1 \cdot 00$ & $1 \cdot 00,1 \cdot 00$ & $1 \cdot 00$ & $1 \cdot 00,1 \cdot 00$ & $1 \cdot 00$ & $1 \cdot 00,1 \cdot 00$ & $1 \cdot 00$ & $1 \cdot 00,1 \cdot 00$ \\
\hline $05.00-10.59$ hours & $1 \cdot 70$ & $1 \cdot 39,2 \cdot 07$ & $1 \cdot 41$ & $0.97,2.06$ & $0 \cdot 84$ & $0.69,1.02$ & $1 \cdot 29$ & $0 \cdot 90,1 \cdot 86$ \\
\hline $11.00-15.59$ hours & 1.55 & $1 \cdot 26,1 \cdot 89$ & $2 \cdot 38$ & $1 \cdot 63,3 \cdot 48$ & $2 \cdot 42$ & $2 \cdot 01,2 \cdot 92$ & $2 \cdot 80$ & $1.97,3.96$ \\
\hline 16.00-22.59 hours & $5 \cdot 73$ & $4 \cdot 70,6 \cdot 99$ & $4 \cdot 53$ & $3 \cdot 15,6 \cdot 52$ & $1 \cdot 42$ & $1 \cdot 18,1 \cdot 71$ & $9 \cdot 90$ & $7 \cdot 00,14 \cdot 01$ \\
\hline
\end{tabular}

ref., reference category; GED, General Educational Development; API, Asian Pacific Islander; Al, American Indian; AN, Alaskan Native.

Multinomial logistic regression models performed the regression of social context (alone, immediate family, extended family, friends/colleagues/others, and multiple categories) v. gender, age, education, race/ethnicity, weight status and time.

All variables were significant at $P<0.01$ except weight status.

normal weight respondents $(\mathrm{OR}=1 \cdot 12(95 \%$ CI $1 \cdot 03$, $1 \cdot 23)$ to $1 \cdot 14(95 \%$ CI $1 \cdot 05,1 \cdot 24)$ ), respondents with a high-school education and above (OR $=1.56$ (95\% CI $1 \cdot 34,1 \cdot 82)$ to $2 \cdot 07(95 \%$ CI $1 \cdot 79,2 \cdot 41))$ and in the middle of the day $(\mathrm{OR}=4 \cdot 35(95 \% \mathrm{CI} 3 \cdot 45,5 \cdot 47))$. Odds ratios for 'other' locations $v$. home/yard were similar to those for eating/drinking in restaurant/bar/retail locations.

Social context was also strongly associated with the distribution of eating/drinking episodes by demographic characteristics (Table 4). Table 4 results present the odds of episodes occurring within social contexts of the immediate family, extended family, friends/colleagues/ others and combinations of these (multiple categories) $v$. alone. Eating/drinking with immediate family was positively associated with age $(\mathrm{OR}=1 \cdot 15(95 \% \mathrm{CI} 1 \cdot 04,1 \cdot 27)$ to $1 \cdot 23(95 \%$ CI $1 \cdot 09,1 \cdot 39))$, education level $(\mathrm{OR}=1 \cdot 16$ (95\% CI $1 \cdot 03,1 \cdot 30)$ to $1 \cdot 36(95 \%$ CI $1 \cdot 21,1 \cdot 54)$ ), obesity $(\mathrm{OR}=1 \cdot 13(95 \% \mathrm{CI} 1 \cdot 04,1 \cdot 22))$, children in the household
$(\mathrm{OR}=3 \cdot 39 \quad(95 \%$ CI $3 \cdot 14,3 \cdot 66))$ and time of day $(\mathrm{OR}=1 \cdot 70(95 \%$ CI $1 \cdot 39,2 \cdot 07)$ to $5 \cdot 73(95 \%$ CI $4 \cdot 70$, 6.99)), but was not associated with gender or employment status except for those not in the labour force (OR $=1.83$ (95\% CI $1 \cdot 67,2 \cdot 00)$ ). In contrast, eating/drinking with extended family was positively associated with being female $(\mathrm{OR}=1.58(95 \%$ CI $1.38,1.81))$ and strongly negatively associated with age $>34$ years $(\mathrm{OR}=0 \cdot 25(95 \%$ CI $0 \cdot 20,0 \cdot 31)$ to $0 \cdot 39(95 \%$ CI 0.32, 0 47)). Younger people were more likely to eat/drink with friends and colleagues or multiple categories. Note that Hispanics consistently reported a greater likelihood of eating with other people compared with Whites, Blacks and other race/ethnic groups $(\mathrm{OR}=1 \cdot 10(95 \% \mathrm{CI} 1 \cdot 00,1 \cdot 22)$ to $1 \cdot 49(95 \% \mathrm{CI}$ $1 \cdot 22,1 \cdot 81)$ ).

Table 5 describes predicted probabilities of eating/ drinking episodes within physical context by social context (i.e. whom the respondent reported they were 
Table 5 Predicted probabilities (PP) for eating/drinking episodes within physical contexts by social context, American Time Use Survey, USA, 2006-2008

\begin{tabular}{|c|c|c|c|c|c|c|c|c|}
\hline & \multicolumn{2}{|c|}{ Home/yard } & \multicolumn{2}{|c|}{ Workplace } & \multicolumn{2}{|c|}{ Restaurant/bar/retail } & \multicolumn{2}{|c|}{ Other } \\
\hline & PP & SE & PP & SE & PP & SE & PP & SE \\
\hline Alone & $0 \cdot 77$ & 0.00 & $0 \cdot 15$ & $0 \cdot 00$ & 0.06 & $0 \cdot 00$ & 0.02 & 0.00 \\
\hline Family & $0 \cdot 87$ & 0.00 & 0.01 & 0.00 & $0 \cdot 11$ & 0.00 & 0.02 & 0.00 \\
\hline Extended family & $0 \cdot 76$ & 0.01 & $0 \cdot 21$ & 0.00 & $0 \cdot 18$ & 0.01 & 0.04 & 0.01 \\
\hline Friends/colleagues/others & 0.25 & $0 \cdot 01$ & $0 \cdot 41$ & $0 \cdot 01$ & $0 \cdot 26$ & $0 \cdot 01$ & 0.08 & 0.00 \\
\hline Multiple categories & $0 \cdot 71$ & 0.01 & 0.01 & 0.00 & $0 \cdot 21$ & 0.01 & 0.07 & 0.01 \\
\hline
\end{tabular}

Multinomial regression models performed the regression of physical context $v$. social context (alone, family, extended family, friends/colleagues/others and multiple categories), gender, age, education, race/ethnicity, weight status and time. Results for gender, age, education, race/ethnicity, weight status and time not shown here because trends are similar to those observed in Tables 4 and 5 .

All predicted probabilities shown were significant at $P<0.01$.

with when eating/drinking in physical contexts). For ease of interpretation, predicted probabilities are reported which are the standardized values that adjust for all covariates in the model. Among eating/drinking episodes occurring at the home/yard, the greatest proportion of episodes occurred with immediate family ( $87 \%$ ), followed by eating alone (77\%) and extended family (76\%). Episodes occurring in the workplace were most common with friends/colleagues/others and extended family, followed by eating alone. Episodes of eating/drinking at a restaurant/bar/retail location were most likely to occur with family/friends/colleagues. Eating/drinking in physical contexts outside the home/yard was more likely to occur with friends/colleagues/others in the workplace (41\%) and in restaurant/bar/retail (26\%) locations.

\section{Discussion}

The present study describes the physical and social contexts of eating/drinking episodes in a nationally representative sample of US adults from 2006-2008. Distinct patterns of eating/drinking episodes within particular contexts were observed in subpopulations by gender, age, education, employment status and presence of children $<18$ years old in the household. These results confirm and extend the concept that everyday eating and drinking episodes can be categorized in multiple dimensions. Location and social setting are two of the eight dimensions explored by Bisogni et $a l$. and the ATUS is a particularly rich and novel source of data to examine these dimensions.

Several major themes emerged regarding the role of physical and social contexts of eating and drinking episodes and next steps for research at a national level. Despite recent attention to increased eating outside the home, the current study finds that most primary episodes continue to occur in the home, especially in women, older adults and those not in the labour force. The high probability of adults reporting episodes of eating with family contributes to evidence supporting development of eating/drinking behavioural interventions within the home and family. Work in this area could build on current literature related to the family as a social context for taste preferences, cues for intake ${ }^{(17)}$, eating patterns and routines $^{(12,18,19)}$. Field studies on food intake report a positive relationship between the number of people that individuals report eating with and the amount of energy consumed $^{(20,21)}$. This finding appears to be consistently reported regardless of whether the meals are eaten at home or away from home, and hunger ${ }^{(22)}$. Bisogni et al. suggest that just as important as the social context, in describing eating episodes, is the role of the particular individual and his/her relationships with others in the social context. These factors can contribute to mental processes, moods and feeling happy when eating with others, and foods may be rated as better tasting ${ }^{(9,22)}$.

Strong associations between eating/drinking episodes and the social and physical dimensions of eating examined with gender, age and presence of children in the household suggest that it might be useful to adopt a lifecourse perspective in characterizing eating behaviour ${ }^{(23)}$. Such a perspective could enrich the Bisogni framework of eating behaviour by embedding their existing dimensions in the life course. For example, trends observed for age groups within social contexts emphasize the changing social networks associated with ageing and greater age. Gender roles and gender stereotypes associated with lifestyles, caretaking responsibilities for children, food preparation, and other familial roles and responsibilities could be factors that may contribute to observed patterns of eating and drinking ${ }^{(24)}$.

Employment places constraints on personal schedules, which may explain the greater probability of eating/ drinking episodes with family for those not employed full-time. Trends highlight the importance of exploring the potential interplay between time of day, daily routines, time spent eating, and physical and social contexts of episodes ${ }^{(9)}$. Most episodes of eating/drinking with family occurred in the evening, which can reflect family meal times. However, findings also identify a subgroup of individuals eating late at night, alone and at home. More information on stimuli within these contexts during late night hours would enhance understanding of occurrence of these episodes. 
Sociological theory and ecological models ${ }^{(12)}$ suggest potential interplay between social and physical contexts on behaviours. Models examining physical by social contexts found a complementary nature, indicating both dimensions should be studied in combination and future studies should continue to examine episodic trends by sociodemographics. Because the present data are crosssectional, it was not possible to determine if physical or social contexts caused reported episodes of eating. Some observed associations seem likely to represent reciprocal causation. For example, social circumstance may cause eating episodes but hunger, social norms or circadian effects lead to social interactions and eating. Thus, the associations reported here are directions requiring future causal analysis using time-intensive measures such as ecologic momentary assessment. Findings from the current study underscore the multiple and overlapping dimensions of eating/drinking episodes and the interaction between these factors to shape how people make choices about what they eat and how this may be situation dependent. As discussed in the Bisogni framework, these combinations of physical and social contexts with other dimensions such as mental processes and time could further delineate and describe patterns of episodes and foods consumed at each episode.

The present study found overweight and obese respondents had a greater odds of reporting an episode of eating in social situations $v$. alone (e.g. immediate family and extended family) as well as episodes occurring in restaurant/bar/retail locations. These findings parallel extant literature highlighting the higher energy content of meals eaten outside the home. Findings regarding the slightly greater social nature of episodes in this population subgroup provide support for consideration of the broader multidimensional nature of eating/drinking episodes, where social meaning and mental processes ${ }^{(9,13)}$ associated with these social contexts (e.g. purpose of meal such as celebration, values) ${ }^{(25)}$, role of the individual and other persons (e.g. parent, host, guest ${ }^{(9,25)}$ and social norms for foods consumed can further influence and characterize an episode ${ }^{(9)}$. Other ATUS studies examining patterns of eating report positive relationships between weight and television viewing activities ${ }^{(14,25)}$, food preparation and clean up, and working ${ }^{(26,27)}$, as well as positive associations between duration of episodes and weight status ${ }^{(28)}$. Cumulatively, these studies ${ }^{(29)}$ highlight the complexity of examining multi-way interactions between the specific contexts highlighted in these results and other dimensions of eating/drinking episodes, particularly mental processes and other activities that may occur along with the consumption of foods/drinks.

There are a number of limitations to the current study. Recall bias and cognitive challenges associated with reporting eating/drinking episodes and context are likely to occur. However, the reliability of self-report in time use surveys has been tested in behavioural research (e.g. physical activity) with high reliability (intra-class correlation $=0 \cdot 73-0 \cdot 74)^{(30)}$. Future studies examining the reliability of $24 \mathrm{~h}$ recall using objective measures of time use in combination with $24 \mathrm{~h}$ diaries would contribute to methodology in this field. A measured BMI is preferred, but differences between self-reported and measured BMI are minimal ${ }^{(31,32)}$ and biases associated with reported height and weight appear to be minimal in the ATUS ${ }^{(27)}$. Because the present study focused on discrete episodes of eating/drinking, it examined primary eating/drinking episodes (which were the only activities reported at a particular time). It did not capture episodes of eating/drinking that occurred concurrent with other activities, such as working or driving, because they were outside the scope of this study. Analyses did not account for seasonality and other temporal factors, but the ATUS design and weighting by month and day of the week allow results to be generalizable to any given day.

Measurement of physical context is also limited to categorizing types of locations. There is also considerable variation in physical context within these locations and some of this variation could help explain differences in eating behaviour. For example, there could be distinct differences in aspects of eating in the home in the dining room, kitchen or in a room with a television. Similarly, in a restaurant, dining at the bar $v$, at a table may have distinct social and behavioural consequences that influence the amount and type of foods served and consumed $^{(9)}$. Despite these limitations, time use data concerning eating behaviour complement other national survey data by placing episodes in a more complete social and physical context at a national level.

Understanding eating/drinking episodes, particularly where and with whom people are eating/drinking, offers important implications for public health researchers, nutritionists and behavioural scientists interested in promoting healthy diets and reducing risks for obesity and other chronic diseases, including cancer. The environment predisposes, enables and reinforces both individual and collective behaviours $^{(33)}$. Thus, identification of contexts for these episodes may have implications for tailored health communication messages and inform intervention efforts by identifying where and when episodes may occur at a population level and the joint relationships between physical and social context on eating/drinking. Additionally, documenting the demographic correlates of the social and physical contexts of eating will allow estimates of the potential scope of interventions designed for particular contexts. Our study highlights the multidimensional nature of eating/drinking behaviour. Further research that can highlight common norms, mental processes and food availability within these contextual situations could identify leverage points for health promotion efforts to support and maintain healthy eating. This type of research would include measurement of context and other situational 
factors characterizing episodes, as well as intake, routine and time.

\section{Acknowledgements}

Sources of funding: This project has been funded in whole or in part with federal funds from the National Cancer Institute, National Institutes of Health, under Contract No. HHSN261200800001E. The content of this publication does not necessarily reflect the views or policies of the Department of Health and Human Services, nor does mention of trade names, commercial products or organizations imply endorsement by the US Government. The National Cancer Institute had no role in the design, analysis or writing of this article. Conflicts of interest: The authors have no conflicts of interest to report. Ethics: Ethics approval was not required. Authors' contributions: A.O. conceptualized the paper, led the analyses and wrote the manuscript; T.E. contributed to paper conceptualization and drafting of the manuscript; G.D. contributed to the drafting of the manuscript and interpretation of the results; F.M.P. reviewed the manuscript; D.B. contributed to the conceptualization and interpretation of the results as well as the discussion and implications of the research.

\section{References}

1. American Institutes for Cancer Research (2007) Food, Nutrition, Physical Activity, and the Prevention of Cancer: A Global Perspective. Washington, DC: World Cancer Research Fund.

2. Stampfer MJ, Hu FB, Manson JE et al. (2000) Primary prevention of coronary heart disease in women through diet and lifestyle. $N$ Engl J Med 343, 16-22.

3. World Health Organization (2003) Diet, Nutrition and the Prevention of Chronic Diseases. WHO Technical Report Series no. 916. Geneva: WHO

4. Guilaumie L, Godin G \& Vezina-Im L (2010) Psychosocial determinants of fruit and vegetable intake in adult population: a systematic review. Int J Behav Nutr Phys Act 7, 12.

5. Shaikh AR, Yaroch AL, Nebeling L et al. (2008) Psychosocial predictors of fruit and vegetable consumption in adults. Am J Prev Med 34, 535-543.

6. Kumanyika SK, Obarzanek E, Stettler N et al. (2008) Population-based prevention of obesity: the need for comprehensive promotion of healthful eating, physical activity, and energy balance: a scientific statement from American Heart Association Council on Epidemiology and Prevention, Interdisciplinary Committee for Prevention (formerly the Expert Panel on Population and Prevention Science). Circulation 118, 428-464.

7. Belk RW (1974) An exploratory assessment of situational effects in buyer behavior. J Mark Res 11, 156-163.

8. Belk RW (1975) Situational variables and consumer behavior. J Consum Res 2, 157-163.

9. Bisogni CA, Falk LW, Madore E et al. (2007) Dimensions of everyday eating and drinking episodes. Appetite $\mathbf{4 8}$, 218-231.

10. Jastran MM, Bisogni CA, Sobal J et al. (2009) Eating routines. Embedded, value based, modifiable, and reflective. Appetite 52, 127-136.
11. Beardsworth A \& Keil T (1997) Sociology on the Menu: An Invitation to the Study of Food and Society. London: Routledge.

12. Delormier T, Frohlich KL \& Potvin L (2009) Food and eating as social practice - understanding eating patterns as social phenomena and implications for public health. Sociol Health Illn 3, 215-228.

13. Blake CE, Bisogni CA, Sobal J et al. (2007) Classifying foods in contexts: how adults categorize foods for different eating settings. Appetite 49, 500-510.

14. Hamrick KS, Andrews M, Guthrie J et al. (2011) How Much Time Do Americans Spend on Food? Economic Information Bulletin no. EIB-86. Washington, DC: US Department of Agriculture, Economic Research Service.

15. US Bureau of Labor Statistics (2012) American Time Use Survey User's Guide. http://www.bls.gov/tus/atususersguide.pdf (accessed February 2012).

16. Korn EL \& Graubard BI (1999) Analysis of Health Surveys. New York: John Wiley \& Sons, Inc.

17. Bellisle F, Dalix AM \& Slama G (2004) Non food-related environmental stimuli induce increased meal intake in healthy women: comparison of television viewing versus listening to a recorded story in laboratory settings. Appetite 43, 175-180.

18. Hughes SO, Power TG, Fisher JO et al. (2005) Revisiting a neglected construct: parenting styles in a child-feeding context. Appetite 44, 83-92.

19. Hughes SO, Power TG, Papaioannou MA et al. (2011) Emotional climate, feeding practices, and feeding styles: an observational analysis of the dinner meal in Head Start families. Int J Behav Nutr Phys Act 8, 60.

20. de Castro JM \& Brewer EM (1992) The amount eaten in meals by humans is a power function of the number of people present. Physiol Behav 51, 121-125.

21. Hirsch E \& Kramer MF (1993) Situational influences on food intake. In Nutritional Needs in Hot Environments, pp. 215-243 [BM Marriott, editor]. Washington, DC: National Academy Press.

22. Bellisle F \& Dalix AM (2001) Cognitive restraint can be offset by distraction, leading to increased meal intake in women. Am J Clin Nutr 74, 197-200.

23. Wethington E \& Johnson-Askew WL (2001) Contributions of the life course perspective to research on food decision making. Ann Behav Med 38, Suppl. 1, S74-S80.

24. Devine CM (2005) A life course perspective: understanding food choices in time, social location, and history. $J$ Nutr Educ Beahv 37, 121-128.

25. Blake CE, Wethington E, Farrell TJ et al. (2011) Behavioral contexts, food-choice coping strategies, and dietary quality of a multiethnic sample of employed parents. J Am Diet Assoc 111, 401-407.

26. Zick CD, Stevens RB \& Bryant WK (2011) Time use choices and healthy body weight: a multivariate analysis of data from the American Time Use Survey. Int J Behav Nutr Phys Act 8, 84.

27. Hamrick KS (2012) Nonresponse Bias Analysis of Body Mass Index Data in the Eating and Health Module. Technical Bulletin no. 1934. Washington, DC: US Department of Agriculture, Economic Research Service.

28. Kolodinsky JM \& Goldstein AB (2011) Time use and food pattern influences on obesity. Obesity (Silver Spring) 19, 2327-2335.

29. Dunton GF, Berrigan D, Ballard-Barbash R et al. (2008) Social and physical environments of sports and exercise reported among adults in the American Time Use Survey. Prev Med 47, 519-524.

30. van der Ploeg HP, Merom D, Chau JY et al. (2010) Advances in population surveillance for physical activity and sedentary behavior: reliability and validity of time use surveys. Am J Epidemiol 172, 1199-1206. 
31. Cawley J \& Burkhauser RV (2006) Beyond BMI: The Value of More Accurate Measures of Fatness and Obesity in Social Science Research. National Bureau of Economic Research Working Paper 12291. http://www.nber.org/papers/w12291 (accessed February 2013).

32. Kuczmarski MF, Kuczmarski RJ \& Najjar M (2001) Effects of age on validity of self-reported height, weight, and body

\section{Appendix 1}

Physical context for eating/drinking episodes, American Time Use Survey, USA, 2006-2008

\begin{tabular}{lrr}
\hline Reported context* & \multicolumn{1}{c}{$n$} & \multicolumn{1}{c}{$\%$} \\
\hline Home/yard & 9378 & $55 \cdot 8$ \\
Workplace & 3001 & $17 \cdot 9$ \\
Someone else's home & 910 & $5 \cdot 4$ \\
Restaurant/bar & 188 & $1 \cdot 1$ \\
Retail location & 333 & $2 \cdot 0$ \\
$\quad$ Grocery store & & \\
$\quad$ Other store/mall & 1264 & $7 \cdot 5$ \\
Car, truck, motorcycle (driver) & 391 & $2 \cdot 3$ \\
Other transportation & & \\
$\quad$ Car, truck, motorcycle (passenger) & & \\
$\quad$ Walking & & \\
$\quad$ Bus & & \\
$\quad$ Subway/train & & \\
$\quad$ Bicycle & & \\
$\quad$ Boat/ferry & & \\
$\quad$ Taxi/limo service & & \\
$\quad$ Airplane & & \\
$\quad$ Other mode of transport & & \\
Other place & & \\
$\quad$ Place of worship, school, outdoors away from & & \\
$\quad$ home, library, other place, bank, gym/health & & \\
$\quad$ lub, post office, unspecified place & & \\
Total & 16801 & $100 \cdot 0$
\end{tabular}

*The American Time Use Survey asks follow-up questions of 'Where were you while you were (activity)?' for activities reported by respondents. Interviewers code responses into one of the twenty-six different categories listed above. The physical context of eating/drinking episodes is defined as 'where' respondents reported they were when eating/drinking. mass index: findings from the Third National Health and Nutrition Examination Survey, 1988-1994. J Acad Nutr Diet 101, 28-34.

33. Sallis J \& Owen N (2002) Ecological models of health behavior. In: Health Behavior and Health Education: Theory, Research, and Practice, 3rd ed., pp. 462-484 [K Glanz, BK Rimer and FM Lewis, editors]. San Francisco, CA: Jossey-Bass.

\section{Appendix 2}

Social context for eating/drinking episodes, American Time Use Survey, USA, 2006-2008

\begin{tabular}{lrr}
\hline Reported context* & $n$ & $\%$ \\
\hline Alone & 15537 & $23 \cdot 2$ \\
Spouse/partner & 15091 & $22 \cdot 6$ \\
Own child & 20918 & $31 \cdot 3$ \\
Own household child & & \\
Own non-household child & 7218 & $10 \cdot 8$ \\
Other family & & \\
Grandchild & & \\
Parent & & \\
Brother/sister & & \\
Other related person & & \\
Foster child & & \\
Parent (not living in household) & & \\
Other non-household family member <18 years & & \\
Other non-household family member >18 years & & \\
Friends & 2503 & \\
Co-workers, colleagues, clients & 2761 \\
Other & & \\
$\quad$ Other non-relative & & \\
Neighbour/acquaintance & & \\
Other non-household children <18 years & & \\
Other non-household adults $>18$ years & & \\
Housemate/roommate & & \\
Roomer/boarder & & \\
Total & & \\
\hline
\end{tabular}

*The American Time Use Survey asks follow-up questions of 'Who was with you/Who accompanied you?' for activities reported by respondents. Interviewers code responses into one of the twenty-two different categories listed above. The social context of eating/drinking episodes is defined as 'whom' respondents reported they were with when eating/drinking. 